\title{
IMPORTANCIA DEL CONTEXTO EN LA COMUNICACIÓN LINGUISTICA
}

\section{Lucía Tobón de Castro*}

En general, se ha considerado que la ciencia es aquella actividad cuyo objetivo es descubrir las leyes o principios que subyace a los hechos o fenómenos que conforman el universo en el cual se halla inmerso el hombre o bien registrar su ocurrencia. Por ello no resulta extraño que, desde el comienzo de su existencia histórica como especie o como individuo, haya sido su preocupación constante desentrañar, bien por curiosidad o por necesidad, aquellos sucesos imposibles de conocer a través de la llamada percepción sensible.

En un comienzo, confirió mayor importancia al entorno natural, porque las necesidades vitales amenazaban su existencia. Fue sólo cuando dominados los peligros ambientales y adquirida conciencia de que él mismo constituía un universo que si bien participaba del mundo biofísico al igual que otros seres, contaba con algo más: el ámbito de la mente que lo tomaba distinto y le pemitía desplazar su atención hacia hechos y fenómenos, producto de su creación, de su invención y de su imaginación, que podían tener o no tener correlato en el medio físico.

Algo semejante a lo logrado por la humanidad a través de su historia, se repite en el individuo que, gracias a procesos experienciales o cognoscitivos, se va adueñando no sólo de cuanto ocurre en su medio natural o social sino de lo que conforma su propio ethos, iniciándose así su interés por escudrinarlo. Por ello no puede resulta extraño que sea el siglo veinte la época de mayor desarrollo de las ciencias del hombre, en general y de la lingüística, en particular, ya que a ella corres ponde ocuparse de entidades tan íntimamente vinculadas a la vida cotidiana como son los códigos verbales ( $\mathrm{y}$ no verbales) presentes en toda comunidad humana.

Establecido así el interés por los comportamientos humanos y de manera particular por la interacción social, es de gran importancia ocupamos de cuál ha sido el valor dado al témino CONTEXTO en el es tudio de la comunicación verbal, a fin de verificar en qué medida ha sido factor determinante en la orientación de los estudios del lenguaje y de sus realizaciones concretas, las lenguas, en todos los momentos del proceso de desarrollo de la lingüística teórica y/o aplicada. Por tanto, debemos comenzar por explicar cuándo y cómo se incorporó la noción de contexto en los distintos modelos creados por los lingüístas y qué cambios semánticos se han efectuado para reflejar las transformaciones conceptuales ocurridas en el interior de la ciencia, para satisfacer así las necesidades de las diversas teorías propuestas hasta ahora.

Para cumplir este propósito, es preciso mencionar el punto de vista de De Saussure, quien en su diseño de un modelo científico de la lengua, quiso desconocer la influencia de que pudieran tener los movimientos de finales del siglo diecinueve, que antecedieron su propuesta renovadora. No obstante, al concebir

\footnotetext{
* Profesora Honoraria de la Universidad Pedagógica Nacional. Profesora e Investigadora del Seminario Andrés Bello. Instituto Caro y Cuervo.
} 
la lingüística como ciencia autónoma, liberada de la filosofía y características básicas de estas etapas precedentes: es tructura del sistema como lo había venido haciendo la gramática tradicional y mantenerse en el nivel descriptivo del comparatismo.

Si bien conoció el pensamiento de Whitney para quien la lengua es una "institución social" cuya razón de existir está en servir de medio de comunicación e instrumento de los procesos de pensar (Mounin:72), con su afimación "la lengua es la foma no la sustancia" (De Saussure: Curso, 171), señala el rasgo que ha definido desde entonces al estructuralismo, cuya teoría ha alejado a la lengua de toda relación con el individuo y con la cultura. Es así como, al ignorar la sustancia deja de lado el problemas del significado, al definir la lengua como el saber que está en la comunidad ignora la creatividad del hablante y al desconocer el uso elimina la presencia del oyente, presentando el acto de comunicación como un hecho psicológico idealizado, sin referencia al acto de habla concreto. En conclusión, toma la lengua como un sistema homogéneo, funcional, que se deja clasificar, desconociendo así las diversidades existentes dentro de todo sistema lingüístico. Por estas razones, no se encuentra en el Curso ninguna referencia al contexto.

Desarrollos ulteriores de la teoría saussureana, que veían la lengua como una red de relaciones funcionales (ver propuestas de Hjemslev y de Martinet), dieron lugar a la introducción del término contexto, entendido como el conjunto de elementos que, en el marco de sus relaciones sintagmáticas pemiten la presencia de unidades lingüísticas que sean compatibles con ellos de acuerdo con las restricciones estructurales que supone cada lengua. Por esto, podemos concluir que el contexto de una unidad Lingüística se puede especificar a base de sus relaciones sintagmáticas (Hjemslev: 53).

Tomando como base las relaciones sintagmáticas (en presencia) y las asociativas (en ausencia) enunciadas por De Saussure, se fomuló el principio de la doble articulación: la primera o de la relaciones sintagmáticas y la segunda o de las relaciones paradigmáticas (Llyons:73, 74). En consonancia, según las posibilidades de aparición cada unidad entra en relación sintagmática con las unidades del mismo nivel y CONSTITUYE EL CONTEXTO. De igual manera, de acuerdo con su aparición en un determinado CONTEXTO entra en relación paradigmática con aquellas unidades que puedan aparecer en el mismo contexto. Veamos algunos ejemplos:

- En el marco de las relaciones sintagmáticas el elemento /p/ en la palabra pan mantiene relaciones con $/ \mathrm{a} / \mathrm{y} / \mathrm{n} /$ con las que aparece y forma el contexto; y de acuerdo con la posibilidad de aparición en el contexto /an/, el elemento /p/ mantiene relación paradigmática con $/ \mathrm{t} / \mathrm{d} / \mathrm{d} / \mathrm{/} / \mathrm{s} / \mathrm{tan}$, dan san y en el elemento $/ \mathrm{n} /$ entra en relación paradigmática con /r/, /z/ par, paz.

- Las relaciones sintagmáticas que son directamente observables, se establecen por contraste. En la expresión un buen bino, existen las que contrae buen con sus vecinos un y vino y las que contrae /n/ con la /i/ precedentes y la /o/ subsiguiente dentro del signo vino.

- Por el contrario las que se dan entre unidades que pueden figurar en el mismo contexto son relaciones de oposición, por cuanto se excluyen. Tal el caso de 
bueno, malo, que pueden aparecer en el mismo contexto, de ahí que donde esté uno de ellos no pueden estar los otros, por tanto en las relaciones paradigmáticas se aplica el procedimiento de substitución (Martinet:68, 35 sgtes). No olvidemos que en materia fonológica Martinet recoge el pensamiento de la Escuela de Praga, expuesto de manera muy clara en los escritos de Trubeztkoy y Jakobson.

Conforme lo han esbozado los discípulos de De Saussure la noción de contexto surgió con una connotación puramente estructural, referida a las restricciones existentes en cada lengua en lo relativo a la manera como se integran las unidades de nivel inferior para formar unidades mayores. Se trata, des de luego, del llamado contexto lingüístico (o conte xto gramatical).

El "distribucionalismo" de Zelling Harris (visión norteamericana del estructuralismo), extiende la influencia de las relaciones sintagmáticas y paradigmáticas al campo del significado y considera que el sentido de la palabra es perfectamente identificable en términos del contexto lingüístico en que ella ocurre; agrega "es posible suponer que cualquier pareja de morfemas que tenga diferente significado, difiera en su distribución" (Harris: 60). Al respecto dice Palmer, "se trata de un punto de vista extremo con relación al contexto lingüís tico, por cuanto la distribución es resultado de la diferencia de significado no causa" (Palmer: 76, 92). Además, esto sería aplicable al nivel sintagmático que es observable, no así al paradigmático cuya relación de substitución con elementos no predecibles, contrariaría el principio del distribucionalismo cuyo objetivo era hallar métodos empíricos que pemitieran deteminar y clasificar los elementos de una lengua.

Algo análogo propuso Firth, al hacer depender el significado de las palabras de las otras formas lingüísticas que las acompañaban, es decir, del puesto que ocupaban dentro del enunciado. En suma, lo importante para Firth no era la distribución ni la referencia al contexto situacional o a otro deteminado contexto, sino lo que primaba era la co-ocurrencia de las formas lingüísticas. De allí su principio de que bastaba hallar la densidad léxica de un texto para comprender el mensaje que portaba.

Esta actitud de explicar el problema del significado mediante las relaciones intralingüísticas y de rechazar, en sus procesos de análisis, la relación de la lengua con el mundo de la experiencia, con los procesos de pensar o con los hechos situacionales, se mantuvo hasta los años sesenta.

Surgió luego un nuevo concepto de contexto, propues to por quienes señalaban nuevas metas a la investigación lingüística, distintas del anális is descriptivo de la estructura de la lengua por la lengua sin vínculos con el usuario o el ambiente, como se venía haciendo dentro del marco estructural.

En primer lugar está la sociolingüística, cuya tarea era el estudio de la lengua en su relación con la es tructura social: bien considerando la estructura social como variable independiente y el cambio lingüístico como variable dependiente 0 tomando ambos factores como codeterminantes y tratando de identificar las correlaciones existentes entre ellos. Para tal fin, debía orientarse la investigación hacia el uso de la lengua, identificar la diversidad lingüística dentro de las 
comunidades y reconocer la influencia del contexto socio-cultural sobre el cambio lingüís tico.

Este enfoque dio lugar a dos tipos de contexto, que aparecen muy bien diferenciados en el modelo socio-semiótico propuesto por Halliday. Tomando como texto "los casos de interacción lingüística en que la gente participa; todo lo que se dice o se escribe en un contexto operativo, en oposición al que aparece registrado en un diccionario o en un libro de ciencia que podría ser considerado un contexto citado" (Hilladay: 78,144), es posible distinguir el contexto de situación y el contexto social. Al igual que Malinoski, entiende el contexto de situación como el conjunto de subsis temas semánticos asociados a un hecho particular y el contexto social con la constelación de significados que conforman la cultura.

Aunque la situación es el entorno en el cual se genera el texto, no puede interpretarse con un sentido de inmediatez en el tiempo o en el es pacio, porque bien puede ocurrir que el contexto de situación no esté presente en el momento de la producción y sea una evocación o un propósito de los participantes. Además, es la estructura social la que define los diversos tipos de contexto social; la que regula los significados y los estilos empleados en las interacciones humanas y, finalmente, es la que crea las jerarquías sociales, los correspondientes registros que las expresan y las normas que regulan el uso de esos regis tros.

Como lo mencioné anteriormente, Malinowski, que ve la lengua desde una perspectiva etnográfica, ve el contexto, tanto físico como cultural de una en enunciado, "como el conjunto de rasgos relevantes del ambiente en que ocurre" (Martín E.H., 85,11 en Etnolingüística: 85,11). Este punto de vista se observa en los antropólogos para quienes existen estos dos conceptos: contexto lingüístico y contexto de situación, porque si cada lengua tiene su propia organización semántica, cada cultura -en la cual está inscrita la lengua- tiene sus situaciones relevantes y específicas. Es así como el potencial combinatorio de cada palabra o emisión está determinado por dos factores: el mensaje y la situación cultural. El mensaje determina el significado contextual o lingüístico y la situación cultural define el contexto situacional.

También se encuentra preocupación por la importancia del contexto en la ciencia de la comunicación. En su obra Enografía de la comunicación (D. Hymes:64), recoge el pensamiento de Sapir cuando en relación con la integración de las ciencias del hombre, dice: "es posible esperar que los lingüístas se den cuenta de la importancia de su disciplina dentro de la ciencia en general y no permanezcan aislados [...] sino le den vida relacionándola con campos de estudio que van más allá de la lengua misma" (Sapir:29). Por eso, al definir las características de la Etnografía de la Comunicación reconoce que no es posible tomar de manera aislada los resultados de la lingüística, la psicología, la sociología o la etnología; es preciso correlacionarlos para hacer el estudio de las lenguas en contextos no lingüísticos, y hallar así pautas de la actividad de hablar. Con marcos de referencia diferentes y tomando la comunidad como contexto bus can descubrir el papel de la lengua en la cultural y en la sociedad. Se sitúa, así, la lengua dentro del proceso de comunicación para analizar la relación entre el mensaje y el contexto, la relación de la lengua tanto con el ambiento natural con social y las habilidades que demuestran los hablantes para seleccionar el código de acuerdo con el evento y la situación pues como anota Bloomfield 
(Language: 62,22 ) es increíble lo variadas que pueden ser las capacidades de los hablantes aún en pequeñas tribus que se presuponen homogéneas.

Esta tendencia a referir el mensaje del texto a factores intrínsecos de índole estructural o extrínsecos de carácter contextual es rechazada por Leech, quien considera "el contextualismo" una instancia innecesaria del análisis del texto (Leech:77).

Has ta aquí me he referido al proceso que, dentro de la lingüística y sus ciencias afines, ha seguido el concepto de contexto y ha sido posible observar cómo se le ha ido substrayendo de un valor puramente formal hacia contenidos de índole semántica, social e incluso cultural. Dicho de otra manera, lo que al comienzo tuvo un marco referencial sumamente restringido, se ha venido aplicando a espacios tan amplios, que abarcan por igual proyecciones de la lengua, fenómenos del campo social y manifestaciones de la cultura, no ya referidos exclusivamente a la naturaleza del código verbal, sino a las interacciones humanas, en las diversas dimensiones en que ellas pueden tener ocurrencia.

Investigaciones recientes han generado nuevos interrogantes que han dado lugar ala revisión de planteamientos en lo relativo la concepción del CONTEXTO. Todo lo que hemos esbozado hasta ahora, ha tratado de4 describir y explicar la función del contexto. Primero, desde el ámbito de la lengua como sistema, de ahí que se le haya situado dentro de la lengua misma y se haya hecho corresponder con la relación funcional de los elementos (visión estructural). Segundo, salido del ámbito del sistema, el contexto fue referido al uso de la lengua en las interacciones humanas y se le identificó con el entorno socio-cultrual que hacía posible la influencia de la sociedad sobre los cambios lingüísticos operados tanto a nivel individual (diafasías), como a nivel colectivo (diatopías y diastratías). Por último, la ciencia de la comunicación lo ubicó en la comunidad de hablantes que, como creadora de redes de interacción humana, determina las relaciones entre código y mensaje y código y usuario.

La anterior síntesis ha puesto de manifiesto que todos los intentos por explicar la relación entre LENGUA y CONTEXTO, han pecado del mismo error. Pretender ubicar, en lo puramente externo las variables que entran en juego, a veces en el sistema mismo y otras, en el sistema y su entorno, cuando en realidad se trata de dos fenómenos que, como dice Chomsky, "están determinados por un factor independiente: la condición humana, la organización de la mente o las demandas intrínsecas de un universo ordenado, que cada quien ha construido y guarda en su cerebro" (Chommsky: 71,47 sgtes).

Por el hecho de centrar su teoría en el estudio de la competencia lingüística, saber que reposa en un hablante genérico, que es más una abstracción que un individuo real, podría considerarse alejado de las preocupaciones del contexto, ya que de manera reiterada afirma que la lingüística (gramática en su terminología) es un estudio que nada tiene que ver con la actuación (uso que cada individuo 
hace de su lengua). Pero no es así, primero, porque su concepción mentalista lo lleva a reconocer las relaciones existentes entre psicología y lingüística, como lo afirma en Lenguaje y entendimiento, 71,10$)$ :

"me parece que la significación que hab rá de tener el estudio del lenguaje reside en [...] que es posible formular de un modo relativamente claro y preciso algunas cuestiones centrales de la psicología [...], cuya contribución más importante al estudio del lenguaje se refiere a la 'inteligencia' que es capaz de procurar en lo que hace relación al carácter de los procesos mentales y de las estructuras que en los mismos se forman y manejan".

$Y$, segundo, porque en su ensayo sobre La naturaleza formal del lenguaje publicado en Fundamentos de la Teoría biológica del Lenguaje, (Lenneberg: 67,397-442) al describir el modelo perceptivo MP, afirma:

[...]"una persona que domina una lengua ha asimilado el sistema de reglas que detemina tanto la conformación fonética de la oración como su contenido semántico intrínseco que llamaremos competencia lingüística... NO ob stante en la actuación participan muchos otros factores. Las creencias extralingüísticas relativas al hablante y la situación. [...]La actuación lingüística está regida por ciertos principios de la estructura cognoscitiva (como limitaciones de la momoria o ciertas situaciones psicológicas, que no son aspectos del lenguaje”.

$Y$ nuevamente, en Lenguaje y entendimiento, al definir competencia lingüística reitera e "aquel sistema de conocimientos y creencias que se desarrollan en la primera infancia y que interactúa con muchos otros factores para deteminar las clases de conducta observadas" (Chomsky: opcit, 4).

Lo anterior, pone de manifiesto que reconoce la existencia de hechos extralingüísticos que intervienen en el proceso de comunicación verbal e influyen en los modelos de producción y comprensión de mensajes por parte de un hablante-oyente real. $Y$ asegura que este tipo de comunicación puede ser analizada desde la doble perspectiva de modelos sensibles o no sensibles al contexto. Con ello plantea su concepción de contexto como factores nolingüísticos, reales o no, presentes en la mente, que influyen en el acto comunicativo, Desafortunadamente no profundizó más en este fenómeno, al dejar de lado el estudio de la actuación.

En el marco de la Teoría del Texto, encontramos un reconocimiento a la importancia del contexto. Al referirse a él, anota Van Dijk, "todo proceso comunicativo es una relación entre dos o más personas que cuentan con elementos comunes que les permiten la comprensión, puesto que utilizan la misma lengua y conocen las convenciones relacionadas con este tipo de interacción. Los interlocutores comparten conocimiento sobre un conjunto de mundos reales o posibles y manejan el aquí y el ahora lógica, física y cognos citivamente". (Van Dijk; 80,273). 
El contexto es sobre todo dinámico, en la medida en que es un transcurso de sucesos que se define por un conjunto ordenado de relaciones en las coordenadas de espacio y tiempo. En él se combinan estructuras concretas, observables y estructuras abstractas que sirven de reguladoras de la interacción. Por ello, no es sólo verbal, ni situacional, es una actividad humana que combina procesos cognitivos, procesos afectivos, actitudes, comportamientos, en fin, todo lo que conforma el pensar y el hacer del individuo.

Existe un conjunto infinito de contextos posibles, uno de los cuales tendrá el status especial de contexto real. El contexto real está limitado por la participación de hablante y oyente en un periodo de tiempo y en un lugar, tiene un principio y un fin que están deteminados por los participantes de acuerdo con la solvencia o las debilidades que éstos manifiesten en cuanto al dominio e integración de saberes metacognitivos y cognitivos aplicables a la situación comunicativa en que se hallan inmersos. El saber metacognitivo supone que cada individuo domine la nomatividad y las convenciones comunicativas de la comunidad a que se pertenece; en tanto que lo cognitivo se refiere a que hablante y oyente tengan estructuras mentales compartidas, relativas a campos del conocimiento; a los factores que definen los estados del contexto real, los participantes posibles, los participantes reales, el conjuntos de actos comunicativos (incluidos los momentos de hablar y escuchar) y otros conjuntos representados por hábitos, creencias, necesidades/des eos, intenciones reales o sentidas, etc.

Según Van Dijk, el objetivo de la pragmática es especificar las relaciones entre TEXTO y CONTEXTO. Para cumplirlo, debe señalar todas las condiciones que permiten la integración de cada texto con su respectivo contexto, habida cuenta de que esta relación se cumple en dos direcciones: ras gos del texto pueden ayudar a construir el contexto y, de igual manera, deteminados rasgos del contexto vgr: creencias, formas convencionalizadas o presencia de la normatividad existente son factores que definen el tipo de texto.

La preocupación por substraer la noción de contexto de lo puramente lingüís tico y de los elementos referenicales que ordean el acto comunicativo, iniciada por Chomsky en su estudio sobre la adquisición y la producción Lingüística y desarrollada por Van Dijk en su teoría del texto, trascendió al campo de la lingüística cognitiva que, al tratar de explicar como jerarquiza y asocia la mente las estructuras conceptuales que va construyendo a partir de experiencia so de procesos cognoscitivos sistemáticos (o asistemáticos), se ha visto obligada a reformular la noción de contexto. Muestras de esta preocupación hallamos en los trabajos de McCawley, Lakoff y Langacker que han venido profundizando en el tema, el producciones conjuntas con especialis tas de ciencia cognitiva.

Tanto McCawley como Lakoff, al sustentar su tesis de que no existe una estructura profunda como elemento básico de los procesos de producción y recepción del mensaje verbal, conceden toda la importancia a las asociaciones de conceptos que, en la mente del hablante-oyente, dan lugar a estructuras 
comunicativas que integran significado-sentido, expresión lingüística y elementos contextuales que ubiquen el mensaje en ejes como real-virtual, tiempo-espacio, punto de referencia etc.

Finalmente, Langaker, en la actualidad dedicado a la investigación sobre la cognición, señala que "uno de los principios de organización de la lingüística cognitiva es negar la autonomía de la estructura lingüística". Asegura, de manera particular, que habilidades cognitivas fundamentales y modelos cognitivos derivados de la experiencia, tienen manifestaciones lingüísticas directas y amplias $y$, a su turno, que la estructura de la lengua proporciona claves muy important4es respecto a los fenómenos mentales básicos. Recientemente la lingüística cognitiva ha encontrado suficiente evidencia para sustentar que la conformación de conceptos como fuerza dinámica, esquemas de imágenes, construcciones objetivas vs subjetivas, y correspondencias entre dominios cognitivos y espacios mentales son fenómenos lingüísticos y no psicológicos como se había sostenido des de el constructivismo.

A partir de las anteriores premisas sustenta el hecho de que cada individuo a medida que construye los esquemas mentales de su discurso va introduciendo los elementos contextuales (lógicos y pragmáticos) que lo configuran y, por ello, no hay que buscar fuera de la mente del hablante todos los factores que ubican un determinado proceso comunicativo en marcos cognos citivos, sociales o culturales. ¿Acaso todos los elementos que constituyen la cultura no conforman una experiencia cognoscitiva que la mente humana va acumulando y categorizando desde la infancia?

Su preocupación respecto a la relación lengua y pensamiento, se manifiesta en el sentido crítico del siguiente comentario: "hemos comprometido todos nuestros esfuerzos en hacer una disección de la lengua para identificar las partes de que consta, los elementos que la forman, sus relaciones, pero es el momento de cambiar el énfasis del sistema mismo, a algunos hechos más importantes: ¿cómo se relacionan lengua y pensamiento? ¿está nuestro pensamiento moldeado por nuestra lengua? ¿podemos pensar sin referir nuestros pensamientos a la lengua que conocemos? Al respecto es posible recordar algunos argumentos esbozados en su teoría:

- Puesto que el pensamiento es una actividad mental, podemos presumir que como tal es anterior a cualquier forma de representación y,por tanto, no llegar siempre a un esquema lingüístico, sino conducir a otro tipo de manifestación (actitud, reacción corporal, desplazamiento espacial, etc.)

- El sistema lingüístico es semejante a la partitura de una sinfonía y tanto en uno como en otra el simple conocimiento de las leyes que regular su funcionamiento no bastan para lograr la comunicación cognos citiva o estética, es preciso el acervo de conocimientos, la creatividad y la originalidad que tanto el hablante como el ejecutante exhiban para que la recepción y la comprensión 
se produzcan. Luego la participación de la mente para generar las bases contextuales es lo más importante. Hemos venido discurniendo sobre la forma como se generó la lingüística, la noción de CONTEXTO y hemos podido apreciar que las distintas concepciones han sido el producto de los modelos lingüísticos aplicados a la descripción y/o explicación del actoverbal. Ahora sólo nos resta comprender la importancia que el contexto encierra en el logro de la comunicación verbal, no como elemento superpuesto ajeno al proceso sino como un esquema cognitivo y/o experiencial que sirve de base a la configuración lógico-semántica del texto/discurso.

Sólo en la medida en que hablante y oyente compartan el contexto como conteniod cognoscitivo y experiencial, serán capaces de descifrar las marcas (o claves) pres entes en los recursos lingü ís ticos utilizados y estarán en capacidad de comprender los mensajes. Es de tal magnitud la importancia de los rasgos contextuales presentes en la mente de los interlocutores que, en muchas oportunidades, permiten subsanar las dificultades de la falta de dominio de una lengua. Cuenta pues, más el 'dominio' del lenguaje que el saber de una lengua.

\section{BIBLIOGRAFÍA}

Bloomfield, L. Language, Holt, Renehart \& Winstob, Inc.:62 Chomsky, N. Lenguaje y Entendimiento. Seix Barral : 73. La naturaleza formal del lenguaje, Wiley \& Sons: 67

Firht, J.R. Papers in Linguistics. Osford University Press: 51 Halliday, MAK. El lenguaje como Simiótica social. Edward Arnold: 78 Harris, Z. Methods in structural linguistics. Chicago: 60 Hjemslev, L. Prolegomena to a theory of Language. International Journal of America Memoir, 7.

Hymes, D. Hacia etnografías de la comunicación. American Anthropologist: 64 Jakobson R. (\& Hall) Fundamentos del Lenguaje. Ayuso: 73

Leech, G. Semántica. Alianza Editorial: 77 Llyons, J. Introducción en Lingüística Teórica. Teide: 73

Malinowski, B. El problema del significado en las lenguas primitivas. Ogden \& Richards: 23

Martín, E. H. Hipótesis acerca del significado contextual. CAEA: Buenos Aires: 85 Matinet, A. Elementos de Lingüís tica General. Gredos: 68

Mounin, G. La Linguis tique du XX siecle. Presses Universitaires de France: 72

Palmer, F. R.Semantics. Canbrudge Ybuversutt Oressm 76

Sapir, E. Language. Harcourt Brace: 49

De Saussure, F. Curso de Lingüística General. Losada: 66

Van Dijk, Texto y Contexto. Cátedra: 80 